\section{Aus Fehlern lernen}

\author{
Man muss kein Prophet sein: 2005 gibt es erst einmal keine Fortsetzung \\ der Ökostever. Die Argumente für eine ökologische Steverreform sind trołzdem \\ aktuell. Deswegen gilt es, aus alten Fehlern zu lernen und neve Strategien \\ zur Durchsetzung des alten Zieles zu entwickeln.
}

$A$ Von Anselm Görres 1 deutschen Autobranche (Stichwort Opel!) müsste ein Politiker schon sehr wagemutig sein, wenn er sich momentan für den flächendeckenden Ausbau der Ökosteuer stark machen wollte. Bundesumweltminister Trittin hält sich in Bezug auf die Ökosteuerfortsetzung schon seit Monaten enorm bedeckt. Die meisten führenden Grünen tun es ihm gleich. Bei der SPD gilt die Ökosteuer zurzeit eher als politisches Selbstmordthema. Auch bei den Bürgern insgesamt hat sie an Zustimmung verloren: Von 2002 an stieg die Ablehnung von 52 auf 58 Prozent. Die Zustimmung sank gleichzeitig von 29 auf 26 Prozent. Fast drei Viertel (73 Prozent) empfinden die ökologische Steuerreform vor allem als sozial ungerecht.

Vor diesem Hintergrund bleibt den Befürwortern der Ökosteuer nur dreierlei zu tun. Erstens, dem politischen Publikum die Erfolge der ersten fünf Ökosteuerstufen 1999 bis 2003 vor Augen führen - schließlich liegen für viele positive Effekte erst kürzlich oder sogar erst demnächst belastbare Auswertungen vor. Zweitens, die fortdauernden Gründe für den Einsatz konsequenter ökologischer Steuerung immer wieder zu betonen. Denn davon, dass Bürger und Politiker Probleme leugnen oder die einzig wirksamen Lösungsinstrumente ablehnen, kommt es leider nicht zu einem Verschwinden der Probleme. Drittens, sich geduldig für den Fall der Fälle vorbereiten. Sowohl aus dem politischen Kalender, aber auch aus aktuellen politischen Debatten können sich immer wieder Handlungsmöglichkeiten ergeben, dem einen oder anderen Element der ökologischen Steuerreform zum Durchbruch zu verhelfen. Kurzfristig dürften sich die größten Chancen bei Flugbenzin bieten, mittelfristig können auch die großen sozial- und fiskalpolitischen Reformdebatten die Tür für Ökosteuer-Fortentwicklungen auf breiterer Front wieder öffnen. Zugleich ist es aber unabdingbar, aus den Kommunikationsfehlern der Vergangenheit zu lernen.
Zugleich ist es wichtig, an den Lehren aus den aktuellen Reformprozessen bei der gesetzlichen Renten- und der Krankenversicherungen anzuknüpfen. Gerade hier lässt sich für Politiker und Bürger eindringlich und nachvollziehbar demonstrieren, dass Verleugnen und Verschleppen der Probleme am Ende nur zu Blamagen, Zeitverlusten und letztlich für alle teureren Lösungen führt.

\section{Denkfehler in der Debatte}

Wir diskutieren über Instrumente meist immer noch wie über Gerichte auf einer Speisekarte, wo man sich das aussucht, was am besten schmeckt. Ökologisch ist das völlig naiv - als ob wir diese Freiheit der Wahl überhaupt noch hätten! In Wahrheit ist es genau wie auch bei der Reform von Renten- oder Krankenversicherung: Wir brauchen jedes einzelne Instrument, und zwar jedes dort, wo es am wirksamsten ist. Nur dann gibt es überhaupt eine Chance, die Probleme zu lösen. Wenn etwa der Wirtschaftminister meint, der Emissionshandel mache die Ökosteuer überflüssig, dann hat er den Umfang des Problems nicht verstanden. Beide Instrumente ergänzen sich hervorragend. Je mehr er den Emissionshandel verwässert, umso mehr Ökosteuer brauchen wir, um die Kyotoziele zu erreichen.

\section{Ernäbrungsalltag und gesellschaftliche Verantwortung}

ist das Thema des Informationsdienstes

\section{Ökologisches Wirtschaften 1/05}

Wenn Sie potenzielle Beiträge haben, wenden Sie sich bitte an die Redaktion.
Den Umweltfreunden ist es bisher kaum gelungen, aus der Debatte um die Zukunft der Krankenversicherung Honig für die Ökosteuer zu saugen. Dabei sieht doch jeder, dass im Augenblick alle Parteien um einen Weg ringen, einen Teil der demographischen Lasten, aber auch der Gerechtigkeitskosten in der Krankenversicherung über höhere Steuerkomponenten zu lösen. Wenn wir weiter die hier bestehenden Chancen verpassen, dann kommt am Ende als einfachste Minimallösung etwas raus, was weder der Umwelt noch dem Arbeitsmarkt nutzt, nämlich nur eine Erhöhung der Mehrwertsteuer.

Wir haben bei der Ökosteuer den Fehler gemacht, nur die schlechte Nachricht in den Vordergrund zu rücken. Alles wird immer teuerer! Wir haben versäumt zu zeigen, dass am Ende des Tunnels Licht kommt: Es ist das Sonnenlicht des Solarzeitalters. Öl, Gas und Kohle müssen nur solange teuerer werden, bis Sonnenenergien wettbewerbsfähig sind. In einigen Bereichen liegt dieser Switching Point gar nicht mehr fern.

Wir müssen endlich lernen, dass in einer entwickelten Volkswirtschaft die Subventionierung von Verkehr zu absurden Fehlentwicklungen führt, nicht nur für die Umwelt. Freie Bürger sollen und dürfen Mobilität und freie Fahrt genießen, aber freie Fahrt gibt es nur, wenn es keine subventionierte Mobilität mehr gibt.

Die Ökosteuer ist für den Verkehr das Gleiche, wie die Praxisgebühr für den Gesundheitssektor: Ein wichtiger Schritt in Richtung Kostenwahrheit und Verursacherprinzip. Dabei gilt es von einem weiteren Denkfehler Abstand zu nehmen. Öffentliche Verkehrsmittel sind nicht immer und automatisch ökologischer als das Auto. Auch Züge brauchen Energie und produzieren Kohlendioxid! Sowohl SPD und Grüne haben in letzter Zeit den Fehler gemacht, die Ökosteuer zu verstecken, fast wie ein behindertes Kind, das man zwar gut behandelt, mit dem man sich aber möglichst nicht auf der Straße sehen lässt. Dabei ist die Ökosteuer ein sehr wohl geratenes Kind, das sich durchaus sehen lassen kann:

- Es ist ein gewaltiger Erfolg, dass mit 75 Milliarden eine Uraltforderung der Industrie erfuillt wurde, nämlich die Deckung der versicherungsfremden Leistungen aus dem Steueretat. Das ist zugleich ein Beitrag zu mehr Verteilungsgerechtigkeit.

- Von den Gegenargumenten der 90er Jahre wurden 90 Prozent durch die tatsächliche Entwicklung widerlegt. Die Ökosteuer hat die Wettbewerbsfähigkeit der Industrie nicht belastet, sondern gestärkt. Die Ökosteuer hat Rentner nicht belastet, sondern 
sogar geholfen, die Renten zu sichern. Die Ökosteuer hat - in Verbindung mit der Einkommensteuerreform - Familien, Haushalte und Schwächerverdienende entlastet, nicht belastet.

\section{Wie kann es weiter gehen?}

Auch Ökosteuerbefürworter lesen Zeitungen und verfolgen den Ölpreisanstieg. Auch wir sehen die Schwierigkeiten, in den harten Zeiten von Hartz noch neue Härten nachzulegen, und seien es auch nur gefühlte Härten mit einem hohen Anteil von Phantomschmerzen.

Angesichts der großen aktuellen Akzeptanzprobleme seien folgende Vorschläge gemacht:

- Dringender Handlungsbedarf besteht beim Schließen der Steuerlücke beim Diesel. Wenn man Benzin und Diesel um 15 Cent verteuert, darf man sich nicht wundern, wenn die Leute auf das um 18 Cent weniger besteuerte Diesel umsteigen. Die Politik darf hier nicht warten, bis auch der letzte Pkw in Deutschland auf Diesel umgerüstet ist. Dann hätten die Autokäufer unterm Strich die Ökosteuer im Verkehr wieder abgeschafft. Wir begrüßen es ausdrücklich, wenn der Finanzminister die Versicherungssteuer abgibt und sich dafür die Bier- und die Kfz-Steuer eintauscht. Dann wären endlich alle emotionalen Flüssigkeiten unter einheitlicher Steuerhoheit. Wir begrüßen es überhaupt nicht, wenn man den Umstieg auf den Partikelfilter wieder subventioniert, wie es zum Beispiel der ADAC fordert. Schrittweise Erhöhung der Dieselsteuer für alle, Kompensation durch eine Reform der Kfz-Steuer, die insbesondere saubere Dieselmotoren entlastet - das wäre intelligente Umweltpolitik!

- Die Reduktion des Mehrwertsteuersatzes für die Bahn ist unter den meisten Umweltverbänden Konsens und wird auch vom Förderverein für ökologische Steuerreform (FÖS) gefordert. Bisher scheiterte sie auch innerhalb der Koalition, nicht zuletzt wegen einiger durchaus ernst zu nehmender Gegenargumente, zum Beispiel der Gefahr ähnlicher Forderungen für Busse und Flugverkehr.

- Angesichts der enormen ökologischen Kosten des Fliegens - wo die gleiche Menge an Kohlendioxid-Emissionen viel größere Schäden anrichtet als auf dem Erdboden - wird die Einbeziehung des Flugverkehrs in die Umwelt- und Umsatzbesteuerung um so dringlicher. Wir bitten die Politiker also: Sorgen Sie für die Einführung einer Kerosinsteuer! Und unterwerfen Sie auch Auslandsflüge der Mehrwertsteuer, natürlich in Zusammenarbeit mit anderen Ländern.
- Je weniger wir momentan mit der flächendeckenden Fortsetzung der Ökosteuer vorankommen, umso wichtiger wird es, wenigstens die offensichtlichsten Schwachpunkte zu reduzieren und vor allem die Ausnahmen abzubauen. Unsere Bitten an die Politik lauten daher: Verbessert und reduziert den Spitzenausgleich für energieintensive Industrien! Er ist ein bürokratisches Monstrum. Wir brauchen hier einen sorgfältig entwickelten Ausstiegspfad, in manchen Bereichen wäre es wahrscheinlich besser, ganz auf die Ökosteuer zu verzichten und stattdessen auf Emissionshandel zu setzen.

Und unterwerfen Sie auch die Kohle der Ökosteuer! Auch wenn wir hier alle wissen, dass dies eine rein symbolische Maßnahme wäre, denn es wird kaum noch mit Kohle geheizt. Aber es ist und bleibt eine Glaubwürdigkeitslücke.

\section{- Verankerung der Debatte}

In schwachen Stunden erfassen auch den konsequenteste Umweltkämpfer tiefe Zweifel am ökologischen Fortschritt der Gesellschaft und der Ökosteuer. Trost und Stärkung lassen sich aber finden, wenn man das Schicksal anderer gesellschaftlicher Reformprozesse betrachtet:

- Beispiel Rente: Jahreslang passierte nichts, obwohl Miegel, Biedenkopf und andere überaus deutlich warnten. Dann führten die einen den Blümfaktor ein und verloren die Wahl. Die anderen schafften ihn erst ab und vier Jahre später unter neuem Namen, als Nachhaltigkeitsfaktor, wieder ein. Wir lernen daraus: Wer harte Reformen als erster einleitet, verliert Stimmen, vielleicht sogar Wahlen. Doch wer sie blockiert, ist letztlich noch mehr blamiert, denn am Ende führt doch kein Weg daran vorbei. Wir können nur hoffen, dass beide politischen Lager in der Lage sind, diese Lehre von der Rente auch auf die Ökosteuer zu übertragen. Vielleicht könnte dann auch Herr Westerwelle einmal von der Rentenerfahrung Einsichten für die Ökosteuer tanken!

- Beispiel Krankenversicherung: Die einen reden von Gesundsheitspauschale, die anderen von Bürgerversicherung. Die einen wollen mehr Eigenbeteiligung der Versicherten, die anderen mehr Kostendämpfung und Effizienz bei den Anbietern. Für den außenstehenden Beobachter ist dabei eigentlich eines ziemlich klar: Wir werden von allem ein bisschen bekommen. Die Probleme in der gesetzlichen Krankenversicherung sind so gewaltig, dass alle Instrumente kombiniert werden müssen, und selbst dann ist noch lange nicht alles im Lot.
- Aus Renten- und Krankenversicherungsdebatte können wir ein Weiteres lernen. Auf der einen Seite ist es wahr, dass „Staatsknete“ die Lösung der Probleme blockieren kann. So hat Oskar Lafontaine die Ökosteuer dazu missbraucht, um notwendige Rentenreformen vor sich her zuschieben. Auf der anderen Seite ist ebenso klar, dass in beiden Systemen ein höherer Anteil von Steuerfinanzierung unverzichtbar ist, um die Beitragszahler zu entlasten und mehr Gerechtigkeit bei den versicherungsfremden Leistungen herzustellen.

- Beispiel Steuerreform: Die Steuerreform der rotgrünen Bundesregierung war nicht nur, nach vielen Jahren des Stillstands, der erste größere Reformschritt im Steuersystem, sie ist in Wahrheit auch sehr gerecht. Die Steuerlast bei den kleinen Einkommen sinkt um über 80 Prozent, bei den großen um weniger als 20 Prozent. Aber die Leute nehmen diese Gerechtigkeit nicht wahr - vielleicht weil die Abschaffung der Vermögenssteuer, aber auch der Einbruch bei den Unternehmenssteuern als ungerecht empfunden werden.

Was lernen wir daraus für die Ökosteuer? Auch hier gibt es eine gefühlte Gerechtigkeitslücke, obwohl die sozialen Effekte der ÖSR gar nicht so schlecht sind. Aber die Politik muss höllisch aufpassen, dass sie nicht die falschen Signale erzeugt, zum Beispiel durch allzu großzügige Ausnahmen. Und sie muss viel mehr tun, um die tatsächlich erreichte Gerechtigkeit zu kommunizieren.

\section{Der Autor}

Dr. Anselm Görres, ist Vorsitzender des Fördervereins Ökologische Steverreform (FÖS).

Kontakt: Förderverein Ökologische Steuerreform, Landsberger Straße 191, 80687 München, Tel. 089-52011313, E-Mail foes@foes.de, www. foes.de 
(c) 20I0 Authors; licensee IÖW and oekom verlag. This is an article distributed under the terms of the Creative Commons Attribution Non-Commercial No Derivates License (http://creativecommons.org/licenses/by-nc-nd/3.o/), which permits unrestricted use, distribution, and reproduction in any medium, provided the original work is properly cited. 